\title{
Electron and Scanning Microscopic Observations on the Syncytiotrophoblast Microvillous Membrane Contribution to Preeclampsia in Early Placental Rats
}

\author{
Manar E Selim ${ }^{1,2 *}$, Nouf G Elshmry ${ }^{3}$ and El Hamidi A Rashed ${ }^{1}$ \\ ${ }^{1}$ Department of Zoology, College of Science, King Saud University, Saudi Arabia, Riyadh-11451, KSA \\ ${ }^{2}$ Department of Zoology, Ain Shams University, Cairo, Egypt \\ ${ }^{3}$ Department of Zoology, College of Science, Hail University, Saudi Arabia
}

\begin{abstract}
Pre-eclampsia is a pregnancy-specific syndrome characterized by new-onset hypertension and proteinuria, occurring usually after 20 weeks' gestation. The current study was carried out on 60 female Wistar rats. Group I: included virgin non-pregnant rats. Group II: included pregnant rats that were received saline solution $(0.5 \mathrm{ml} / 100$ $\mathrm{g}$ body weight) subcutaneously daily starting from day 7 to day 14 of gestation and served as control group. Group III: included pregnant rats that were treated with bestatin dissolved in saline in a dose of $(40.0 \mu \mathrm{g} / \mathrm{ml}) / 100$ $\mathrm{g}$ body weight subcutaneously and daily starting from the same day of gestation and for the same duration as mentioned for group II, to make an animal model of preeclampsia. Hence several possible mechanisms of the activation in pre-eclampsia can be considered, all dependent on the syncytiotrophoblast microvillous surface membrane which is the placental surface in contact with maternal blood. Electron microscopic study revealed regions of syncytiotrophoblast in process of degenerative change were found. These regions were more abundant in pre-eclamptic specimens. Increase irregular deeply indented nuclei of the syncytiotrophoblast layer. Regions of syncytiotrophoblast revealed dilated cisterns of rough endoplasmic reticulum. The objectives of this study were to develop an approach to definitively identify and distinguish the syncytiotrophoblast ultrastructural changes to unambiguously determine the relative susceptibility to constitutive and placental oxidative stressinduced preeclampsia.
\end{abstract}

Keywords: Syncytiotrophoblast; Preeclampsia; Placenta; Pregnancy

\section{Introduction}

The placenta undergoes growth as well as morphological and functional changes with advancing primate pregnancy to promote fetal development. Placental growth results from the proliferation of stem cell-like villous cytotrophoblasts and their morphological differentiation and recruitment into the syncytiotrophoblast [1]. The syncytiotrophoblast is the epithelial covering of the highly vascular embryonic placental villi, which invades the wall of the uterus to establish nutrient circulation between the embryo and the mother. It is a unique tissue in that it is a multi-nucleated, terminally differentiated syncytium that lacks proliferative capacity and instead is maintained by fusion of underlying cytotrophoblast cells [2]. It is the outer syncytial layer of the trophoblasts and actively invades the uterine wall, rupturing maternal capillaries and thus establishing an interface between maternal blood and embryonic extracellular fluid, facilitating passive exchange of material between the mother and the embryo [3].

The syncytiotrophoblast secretes progesterone in addition to human chorionic gonadotropin (hCG) and human placental lactogen (HPL); hCG prevents degeneration of the corpus luteum. Progesterone serves to maintain the integrity of the uterine lining and, until the syncytiotrophoblast is mature enough to secrete enough progesterone to support pregnancy (in the fourth month of embryonic development), it is aided by the corpus luteum graviditatis [2]. Damage of the placenta resulting from ischemia-reperfusion is important to the pathophysiology of preeclampsia. Preeclampsia is a disease of human pregnancy characterized by a systemic maternal inflammatory response associated with endothelial dysfunction, hypertension, and proteinuria. This condition affects 5 to $7 \%$ of all pregnancies and is the main cause of perinatal mortality and morbidity in developed countries. There is also evidence that the risk of subsequent cardiovascular disease is significantly increased in women affected by preeclamptic pregnancies
$[2,4]$. Although the pathophysiology of preeclampsia has not been fully defined, there is evidence that placental oxidative stress attributable to abnormal uteroplacental blood circulation plays a critical role. In preeclampsia, the transformation that normally leads to spiral arterioles with large diameters is defective, and consequently, placental perfusion is compromised [3,4]. Furthermore, it has been suggested that uteroplacental blood flow in preeclampsia is intermittent or pulsatile, likely attributable to the persistent sensitivity of the maladapted spiral arterioles to maternal vasopressin molecules [5]. It has been postulated that the abnormally decreased and intermittent perfusion of the intervillous space of the placenta results in oxidative damage and the release of apoptotic and aponecrotic placental tissue into the maternal circulation [6]. The presence of large amounts of syncytiotrophoblast micro fragments in the maternal circulation is thought to promote the maternal systemic inflammatory response and endothelial dysfunction characteristic of preeclampsia [7]. Indeed, an increased prevalence of apoptotic nuclei has been reported in the syncytiotrophoblast of placentas from pregnancies complicated by preeclampsia [8]. Using an in vitro model of hypoxia/reoxygenation $(\mathrm{H} / \mathrm{R})$ that replicates the oxidative stress in placental tissues undergo during preeclampsia,

*Corresponding author: Manar E Selim, Department of Zoology, College of Science, King Saud University, Saudi Arabia, Riyadh-11451, KSA, E-mail: manar.selim@hotmail.com

Received January 24, 2013; Accepted March 27, 2013; Published March 31 2013

Citation: Selim ME, Elshmry NG, Rashed EHA (2013) Electron and Scanning Microscopic Observations on the Syncytiotrophoblast Microvillous Membrane Contribution to Preeclampsia in Early Placental Rats. J Blood Disorders Transf 4:137. doi:10.4172/2155-9864.1000137

Copyright: ( 2013 Selim ME, et al. This is an open-access article distributed unde the terms of the Creative Commons Attribution License, which permits unrestricted use, distribution, and reproduction in any medium, provided the original author and source are credited. 
Hung et al. [3] demonstrated that the syncytiotrophoblast of normal chorionic villi exposed to H/R undergoes apoptotic and aponecrotic changes similar to those observed in the syncytiotrophoblast of chorionic villi from preeclamptic pregnancies. The syncytiotrophoblast (STB) covering the chorionic villi make up the major surface of direct contact between the placenta and maternal blood. Although it is not known where in the placenta the factor or factors that cause the maternal syndrome originate, in view of its position and size, the role of the syncytiotrophoblast in the pathogenesis of pre-eclampsia needs to be considered. The little that is known about the STB in preeclampsia is derived more from histopathological, than biochemical studies. Changes in the STB include focal loss of microvillus, and dilation of the rough and smooth endoplasmic reticulum, observed with electron microscopy $[3,4]$. These syncytial changes are associated with proliferation of underlying villous cytotrophoblast. Chorionic villi from normal placentae cultured under hypoxic conditions show similar histological changes, raising the possibility that placental ischemia has a role in the pathogenesis of pre-eclampsia $[5,6]$. In our investigation, we try to open the door to more insights by scanning and electron microscopic observations in syncytitrophoblast microvillus membrane of placentas from pregnancies complicated with preeclampsia in rats.

\section{Materials and Methods}

The current study was carried out on 60 female Wistar rats supplied by Medical College animal house at King Khalid University Hospital (King Saud University). Their average weight was 200-250 g. They were 12-16 weeks old. They were housed in a controlled environment and get free access to water ad labitum. Two or three cycling female rats were housed with a male for 24 hours. The presence of sperms in vaginal smears was considered as day 1 of pregnancy. Rats were divided into two groups (20 rats each) according to the following experimental design: Group I: included virgin non-pregnant rats. Group II: included pregnant rats that were received saline solution $(0.5 \mathrm{ml} / 100 \mathrm{~g}$ body weight) subcutaneously daily starting from day 7 to day 14 of gestation and served as control group. Group III: included pregnant rats that were treated with bestatin dissolved in saline in a dose of $(40.0 \mu \mathrm{g} / \mathrm{ml}) / 100$ g body weight subcutaneously and daily starting from the same day of gestation and for the same duration as mentioned for group II, to make an animal model of preeclampsia via induction of placental apoptosis [8]. On day 14 of gestation i.e. 7 days after saline injection in group II or after bestatin treatment in group III, the following parameters were measured in all groups:

1. Mean arterial blood pressure (MAP) from rat-tail by blood pressure system model IITC Biotech Lenfant [9].

2. Some renal function tests including urine volume, creatinine clearance as a measure of glomerular filtration rate (GFR) and $24 \mathrm{hrs}$ urinary albumin excretion using an Agilent/Hewlett Packard 1100 HP series based on size-exclusion HPLC Lenfant [9].

The fetuses and placentas will be removed under anesthesia on day 14 from the early pre-eclamptic group with corresponding control group to manifest pup weights in these two studied groups.

3. The placental samples were removed for the following:

\section{Conventional transmission-electron microscopy}

Tissue placentas were fixed for more than $8 \mathrm{~h}$ at $22^{\circ} \mathrm{C}$ by incubation in a mixture of $2.5 \%$ glutaraldehyde and $2 \%$ paraformaldehyde in $0.1 \mathrm{M}$ sodium cacodylate buffer $\left(\mathrm{pH} 7.4\right.$ ) containing $0.05 \% \mathrm{CaCl}_{2}$. Subsequently, the tissue was post fixed for $1 \mathrm{~h}$ at $4^{\circ} \mathrm{C}$ in $2 \%$ osmium tetroxide in $0.1 \mathrm{M}$ sodium cacodylate buffer ( $\mathrm{pH}$ 7.4) containing $1.6 \%$ potassium ferrocyanide and then rinsed three times in distilled water before dehydration in a graded series of ethanol. The specimens were treated with propylene oxide and embedded in Epon 812 resin. Thin sections were cut, mounted on formvar-coated grids, and contraststained with lead citrate and uranyl acetate before examination under a JEM-1010 (JEOL Ltd., Akishima, Japan) transmission-electron microscope.

\section{Scanning-electron microscopy with $\mathrm{NaOH}$ treatment}

Tissue placentas were fixed as described above. Cryostat sections (thickness, 20-50 $\mu \mathrm{m}$ ) on 2\% 3-aminopropyltriethoxy silane-coated cover slips also were prepared as described above. The sections were then fixed again with $2 \%$ glutaraldehyde in PBS at $4^{\circ} \mathrm{C}$ overnight in a 24 well plate (Sumitomo Bakelite Co., Tokyo, Japan), and immersed in a 6 $\mathrm{N} \mathrm{NaOH}$ aqueous solution for $20 \mathrm{~min}$ at $60^{\circ} \mathrm{C}$ to remove extracellular matrix components, such as collagen fibrils and basal lamina [10] After $\mathrm{NaOH}$ treatment, the samples were washed three times in PBS. Because most placental sections came off the cover slips during the PBS wash, they were carefully collected into $1.5-\mathrm{ml}$ micro centrifuge tubes. The sections were conductive-stained with $2 \%$ osmium tetroxide for $1 \mathrm{~h}$ at $4^{\circ} \mathrm{C}$, rinsed in distilled water, dehydrated through a graded series of ethyl alcohol, treated with $t$-butyl alcohol, frozen for $10 \mathrm{~min}$ at $4^{\circ} \mathrm{C}$, and then evaporated in a vacuum chamber [11]. The freeze-dried samples were mounted on aluminum stubs with adhesive carbon tape, coated with platinum-palladium in a Hitachi E102 ion sputter coater, and then observed in a Hitachi S-3000N scanning-electron microscope at an accelerating voltage of $15 \mathrm{kV}$.

\section{Statistical Analysis}

The data were expressed as the mean \pm S.E. and were analyzed by means of one-way analysis of variance (ANOVA). Statistical evaluation of data was done following Student's $t$-test. A difference was considered significant at $p<0.05$.

\section{Results}

\section{Mean arterial blood pressure (MAP) ( $\mathrm{mmHg}$ )}

As shown in table 1 and figure 1, MAP was significantly increased $(\mathrm{p}<0.05)$ in Bestatin treated pregnant rats (on days 14 as compared to controls and normal non- pregnant rats (129.9 \pm 7.06 vs. $96.4 \pm 4.49$ and $87.15 \pm 2.45, \mathrm{mmHg}$ respectively).

\section{Creatinine clearance $(\mathrm{ml} / \mathrm{min})$}

As shown in table 1 and figure 2 treatment of rats with Bestatin significantly lowered the mean creatinine clearance $(\mathrm{p}<0.05)$ on day 14 of gestation as compared to control and non- pregnant rats (1.002 \pm 0.0132 vs. $2.714 \pm 0.421$ and $2.811 \pm 0.379 \mathrm{ml} / \mathrm{min}$, respectively).

\begin{tabular}{|c|c|c|c|c|}
\hline Groups & MAP (mmHg) & Creatinine clearance (ml/min) & Urine volume ml/24 hr) & Urinary albumin excretion (mg/dl) \\
\hline Group I & $87.15 \pm 2.45$ & $2.811 \pm 0.379$ & $7.994 \pm 1.622$ & $25.30 \pm 1.856$ \\
\hline Group II & $96.4 \pm 4.49$ & $2.714 \pm 0.421$ & $6.818 \pm 1.125$ & $24.62 \pm 1.478$ \\
\hline Bestatin-treated group & $129.9 \pm 7.06^{*}$ & $1.002 \pm 0.0132^{*}$ & $5.291 \pm 0.214^{*}$ & $2.001 \pm 0.143$ \\
\hline
\end{tabular}

*Significant changes at $p<0.05$

Table 1: Mean values $\pm S D$ of the measures parameters in non- pregnant rats, control none treated pregnant rats and treated pregnant rats. 
Citation: Selim ME, Elshmry NG, Rashed EHA (2013) Electron and Scanning Microscopic Observations on the Syncytiotrophoblast Microvillous Membrane Contribution to Preeclampsia in Early Placental Rats. J Blood Disorders Transf 4:137. doi:10.4172/2155-9864.1000137

Page 3 of 8

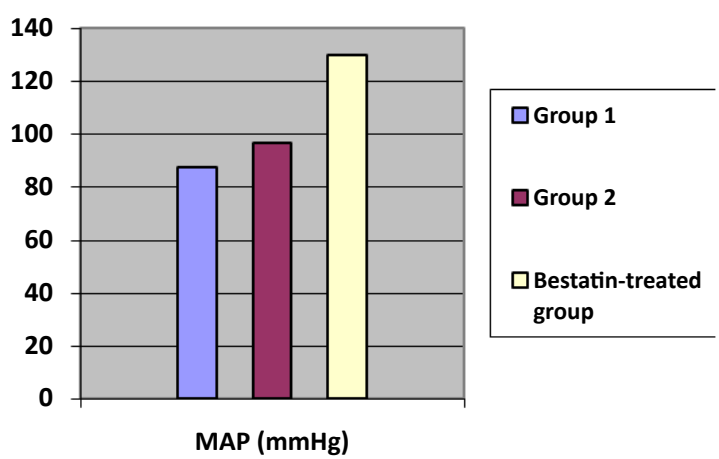

Figure 1: Mean values $\pm \mathrm{SD}$ of the mean arterial blood pressure $(\mathrm{mmHg})$ in the studied groups.

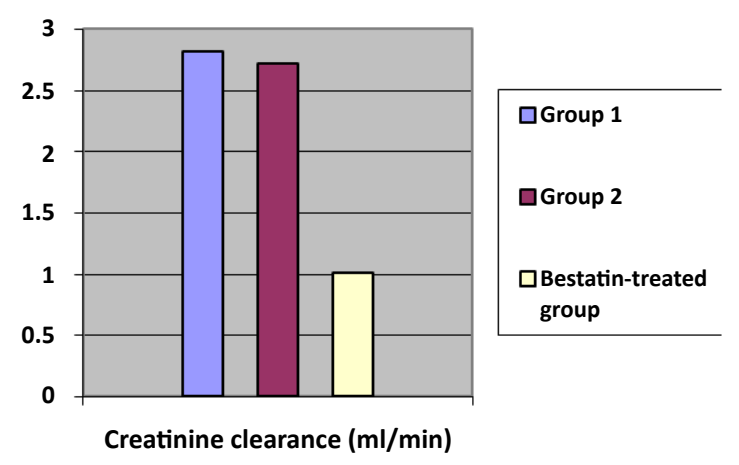

Figure 2: Mean values $\pm \mathrm{SD}$ of creatine clearance $(\mathrm{ml} / \mathrm{min})$ in the studied groups.

\section{Urine volume ( $\mathrm{ml} / 24 \mathrm{hrs})$}

As shown in table 1 and figure 3 , a significant decrease $(\mathrm{p}<0.05)$ in urine volume was detected in Bestatin treated pregnant rats on day 14 as compared to controls and non - pregnant rats (5.291 \pm 0.214 vs. $6.818 \pm 1.125$ and $7.994 \pm 1.622 \mathrm{ml} / 24 \mathrm{hrs}$, respectively).

\section{4 hrs urinary albumin excretion ( $\mathrm{mg} / \mathrm{dl})$ (Table 1)}

As shown in table 1 and figure 4 urinary albumin excretion was significantly increased $(\mathrm{p}<0.05)$ in Bestatin as compared to controls and non- pregnant rats $(123.89 \pm 3.186$ vs. $24.62 \pm 1.478$ and $25.30 \pm$ $1.856 \mathrm{mg} / \mathrm{dl}$, respectively).

\section{Pup weight (g)}

As shown in table 1 and figure 5, pup weight was significantly decreased $(\mathrm{p}<0.05)$ in Bestatin treated pregnant rats as compared to normal pregnant rats $(1.034 \pm 0.361$ vs. $2.001 \pm 0.143 \mathrm{~g})$.

\section{Electron and Scanning Electron Microscopic Observations}

\section{Control placenta}

The syncytiotrophoblast, the outermost lining of the terminal villi, was continuous with each other. The nucleus was irregular in shape and darkly stained with condensed chromatin material inside it (Figure 6).

The syncytiotrophoblast (STB) layer, which is in contact with maternal blood in the maternal intervillous spaces, has numerous short microvilli projecting into the intercellular spaces (Figures 6 and 7). The cytoplasm of syncytiotrophoblast was darkly stained, with numerous electron-dense granules of uniform size with rough endoplasmic reticulum and mitochondria were clearly seen (Figure 7).

The scanning electron microscope revealed the corresponding pictures of the chorionic villous tree are shown in (Figure 8). These villous structures were characterized by a dense population of multiple small buds like villi containing projections, similar in size and shape, showing smooth and regular framework of the normal villi corresponding to sinusoidal dilatations at the tips of terminal capillary loops.

\section{Preeclamptic placenta}

Presence of villous trophoblast with degenerative changes

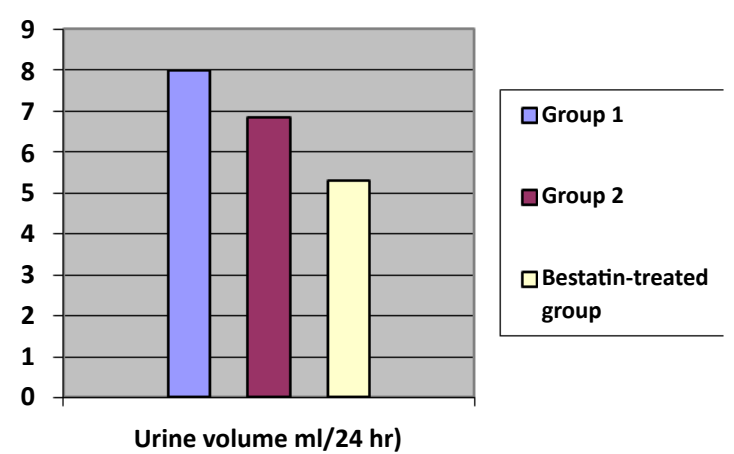

Figure 3: Mean values \pm SD of urine volume $(\mathrm{ml} / 24 \mathrm{~h})$ in the studied groups.

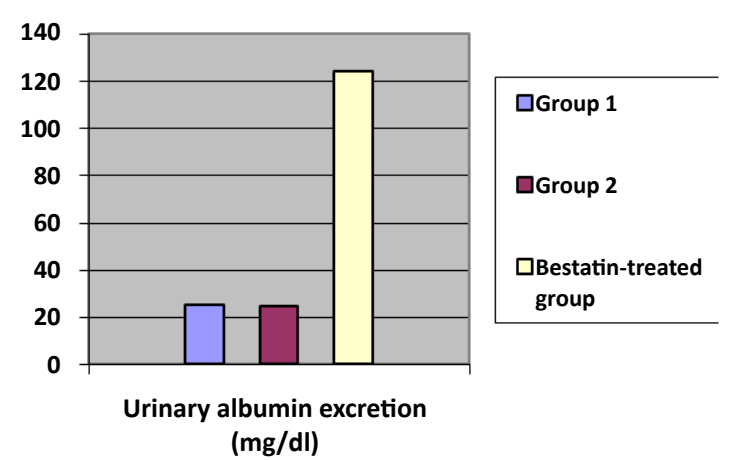

Figure 4: Mean values \pm SD of urinary albumin excretion $(\mathrm{mg} / \mathrm{dl})$ in the studied groups

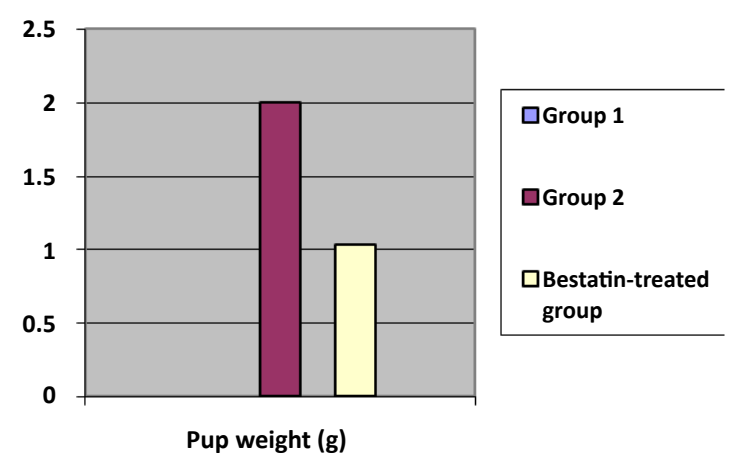

Figure 5: Mean values \pm SD of pup weight $(\mathrm{g})$ in the studied groups. 
Citation: Selim ME, Elshmry NG, Rashed EHA (2013) Electron and Scanning Microscopic Observations on the Syncytiotrophoblast Microvillous Membrane Contribution to Preeclampsia in Early Placental Rats. J Blood Disorders Transf 4:137. doi:10.4172/2155-9864.1000137

Page 4 of 8

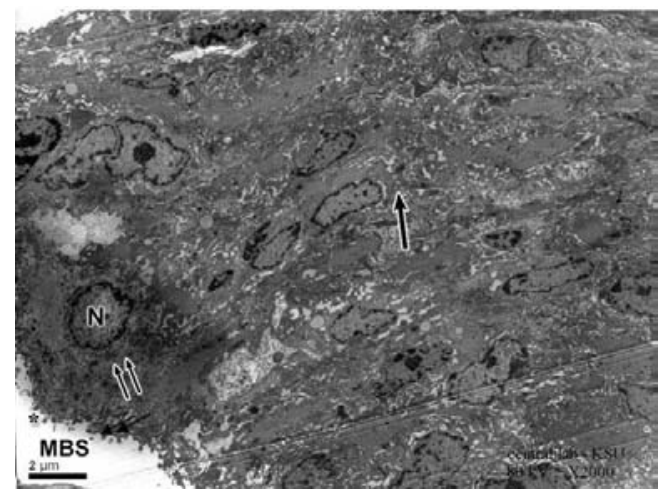

Figure 6: Electron micrograph of control placenta showing a nest of proliferative cytotrophoblast $(\uparrow)$ covered by a layer of syncytiotrophoblast $(\uparrow \uparrow)$ that lines a maternal blood space (MBS). Note the nucleus(N) and short microvilli(* (of syncytiotrophoblast . X2000.

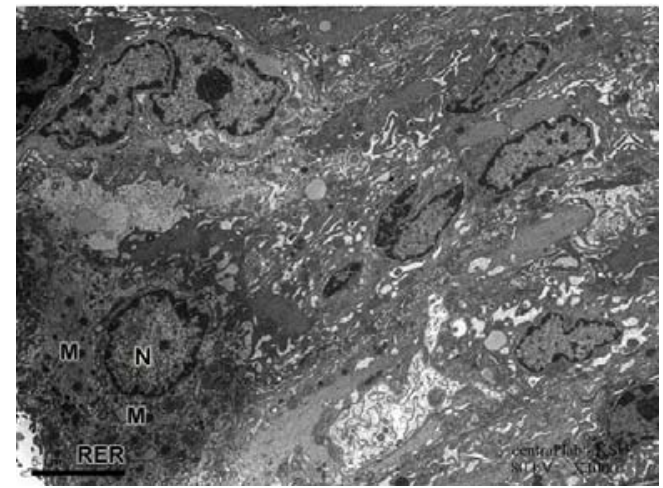

Figure 7: Enlarged part of fig.1 showing detail of cellular and syncytia trophoblast in this region. Notice the nucleus of syncytiotrophoblast, rough endoplasmic reticulum RER and mitochondria(M)are clearly seen . x4000

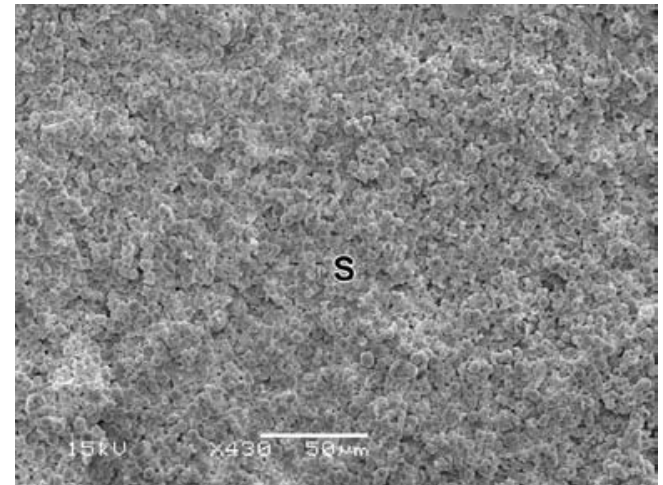

Figure 8: A scanning electron micrograph of the normal chorionic villi showing smooth and regular framework of the normal villi. $\times 430$.

surrounding the peripheral area of placental villi was put into evidence. Electron microscopic study revealed regions of syncytiotrophoblast in process of degenerative change were found. These regions were more abundant in preeclamptic specimens. Increase in irregular deeply indented nuclei of the syncytiotrophoblast layer (Figure 10,17 and 19). Regions of syncytiotrophoblast revealed dilated cisterns of rough endoplasmic reticulum (Figures 9,10 and 16). This organelle presented degranulation of ribosomes in its membranes. This was not seen in controls. Local loss of microvilli on the free syncytial surface was noticed in some preeclamptic specimens (Figures 1719]. While the free syncytial surface of other specimens appeared with shortening and decreased number of microvilli (Figures 11 and 12) or enlarged rounded or elongated apical microvilli (Figures 14 and 15). Large areas of degenerating syncytiotrophoblast appeared to be delivered to the intervillous space (Figure 13). Fragments of syncytiotrophoblast were expulsed to the maternal circulation (Figure 13). Process of syncytiotrophoblastic edematous change appeared

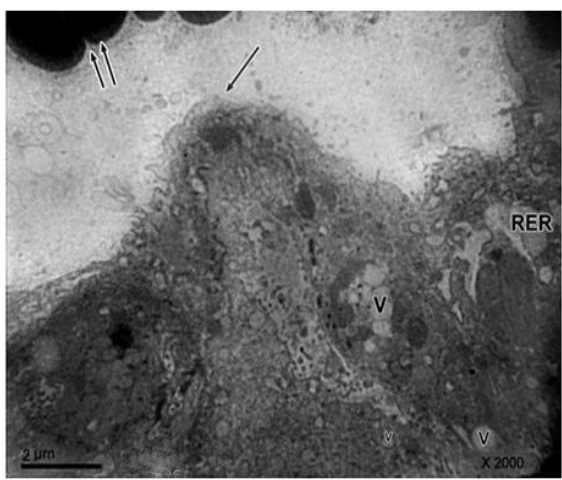

Figure 9: Electron micrograph of a portion of a villous from preeclamptic placenta 14 days showing the maternal erthrocytes $(\uparrow \uparrow)$ and irregularity of syncytial covering borders $(\uparrow)$.Notice the syncytiotrophoblast appeared with vacuolar cytoplasm (V)and dilated R.E.R.X2000.

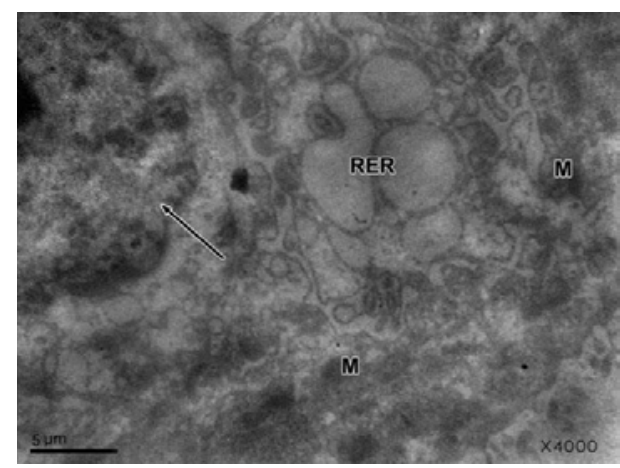

Figure 10: Electron micrograph of a portion of a villous from preeclamptic placenta 14 days showing irregular contour of the nucleus $(\uparrow)$. Notice dilated R.E.R and obscured mitochondria (M).X4000.

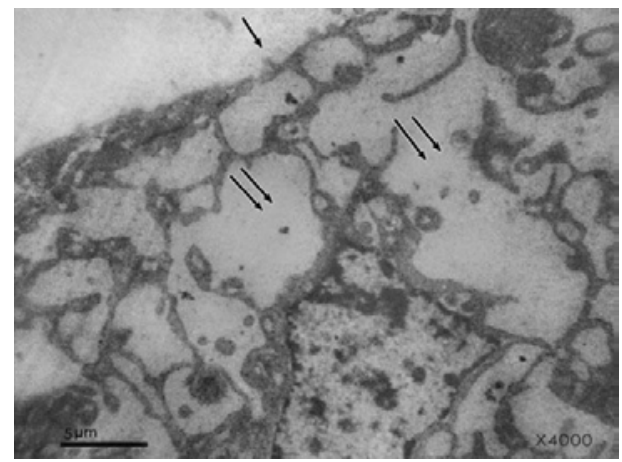

Figure 11: Electron micrograph of a portion of a villus from preeclamptic placenta 14 days showing the syncytiotrophoblat zone appear with very few microvilli $(\uparrow)$ and intracellular oedema $(\uparrow \uparrow)$. X4000 
Citation: Selim ME, Elshmry NG, Rashed EHA (2013) Electron and Scanning Microscopic Observations on the Syncytiotrophoblast Microvillous Membrane Contribution to Preeclampsia in Early Placental Rats. J Blood Disorders Transf 4:137. doi:10.4172/2155-9864.1000137

Page 5 of 8

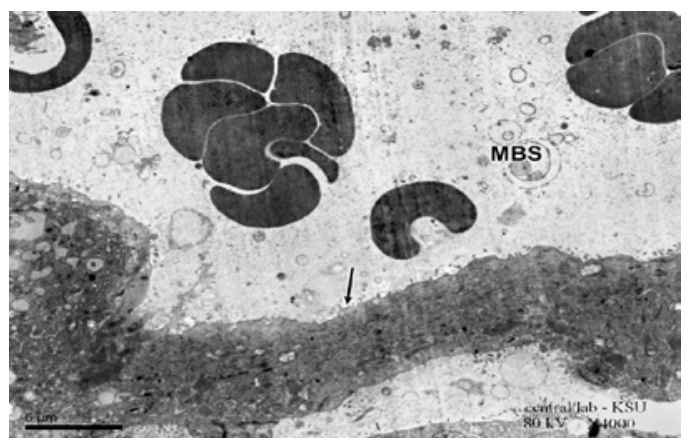

Figure 12: Electron micrograph of a portion of a villous from preeclamptic placenta 14 days showing the maternal blood space (MBS), or intervillous space, is lined by a thin layer of degenerating syncytiotrophoblast with shortening and decreased number of microvilli $(\uparrow)$. X4000.

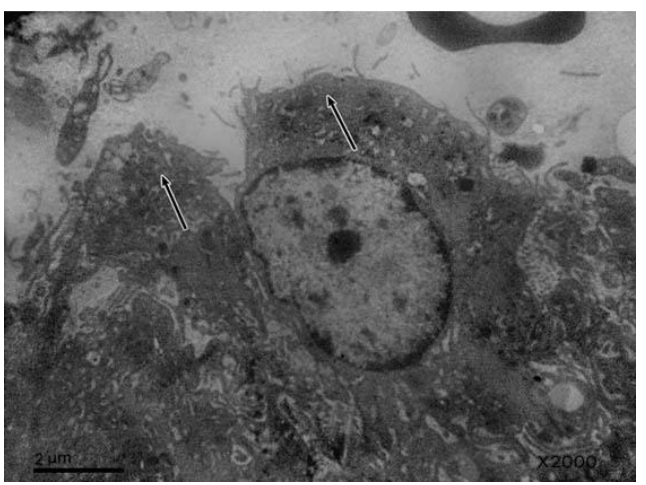

Figure 13: Electron micrograph of a portion of a villous from preeclamptic placenta 14 days showing sloughing part of syncytiotrophoblast to the lumen $(\uparrow) . X 2000$.

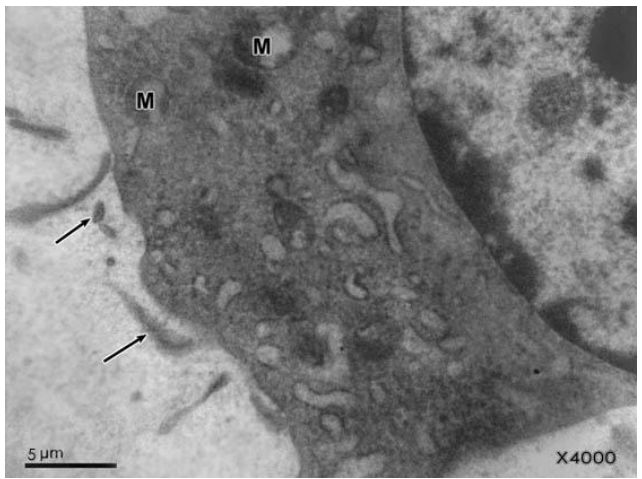

Figure 14: Electron micrograph of a portion of a villous from preeclamptic placenta 14 days showing syncytiotrophoblast with little number of rounded, enlarged microvilli $(\uparrow)$ and destructed mitochondria (M).X4000.

(Figure 11). A syncytiotrophoblastic zone has been detached from the syncytiotrophoblast (Figure 16). There are zones of syncytiotrophoblast cells that contain two types of mitochondria, ones are of clear mitochondrial matrix and others of electron dense mitochondrial matrix observed in degenerating trophoblast cells (Figure 18). The syncytiotrophoblast also showed swollen mitochondria that may strongly with intramitochondrial granules. These mitochondria had interrupted cristae and exhibited vacuolar zone in their light matrix (Figure 14), whilst the controls images of mitochondria preserved the normal ultrastructure. The syncytiotrophoblast cells of the affected villi presented vacuoles of different sizes and shapes (Figure 9), not observed in most of control specimens. Increased deposition of collagen fibers was observed in pre-eclamptic specimens (Figure 20). Scanning electron microscopic examination (Figures 21 and 22) revealed that the chorionic villi of the pre-eclamptic placenta appear irregular than that of normal placenta. The fibrin skeleton of the affected chorionic villi was thickened; rough and irregular (Figure 21). The affected placenta showed multiple chorionic infarcts and many abruptions (Figure 22).

\section{Disscussion}

As previously discussed, a key event in normal placentation is invasion of the maternal spiral arteries in the decidua and myometrium by fetal trophoblast [12]. Zhou et al. [13] reported that this process is defective in pre-eclamptic placentas. Normally trophoblast cells transform from an epithelial phenotype to an endothelial phenotype as they invade the maternal deciduas and myometrium in a process termed pseudo-vasculogenesis. The migrating trophoblasts transform the maternal spiral arterioles that supply maternal blood to the placenta from small caliber resistance vessels to large caliber capacitance vessels allowing adequate maternal blood flow to the placenta. In preeclampsia this process is disordered and the fetal trophoblasts fail to properly invade the maternal myometrium and spiral arterioles [10].

Early placental development occurs in a hypoxic environment

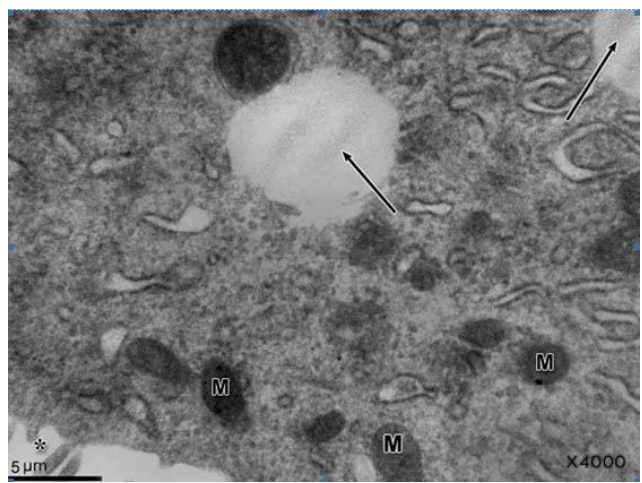

Figure 15: Electron micrograph of a portion of a villous from preeclamptic placenta 14 days showing mitochondria with obscured cristae (M )and the microvilli are rounded or elongated $\left({ }^{*}\right)$. Notice fat droplets are clearly seen ( $\uparrow$. X4000.

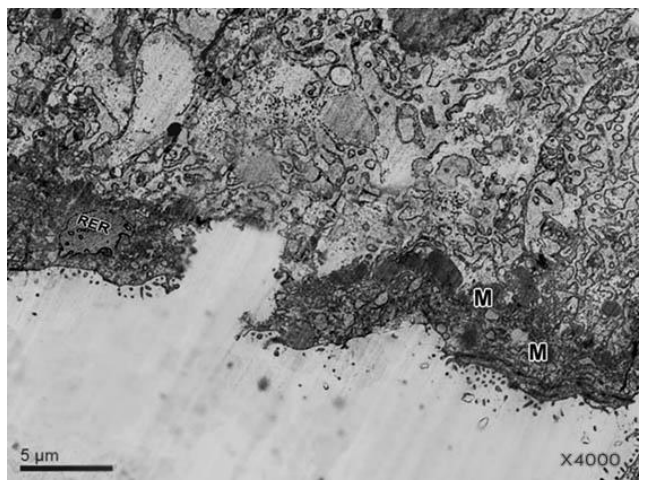

Figure 16: Electron micrograph of a portion of a villous from preeclamptic placenta 14 days showing detachment of syncytiotrophblast from the surface of the placental villi $(\uparrow)$. Electron dense mitochondria $(M)$ and dilated R.E.R of syncytiotrophblast are noticed.X4000. 
Citation: Selim ME, Elshmry NG, Rashed EHA (2013) Electron and Scanning Microscopic Observations on the Syncytiotrophoblast Microvillous Membrane Contribution to Preeclampsia in Early Placental Rats. J Blood Disorders Transf 4:137. doi:10.4172/2155-9864.1000137

Page 6 of 8

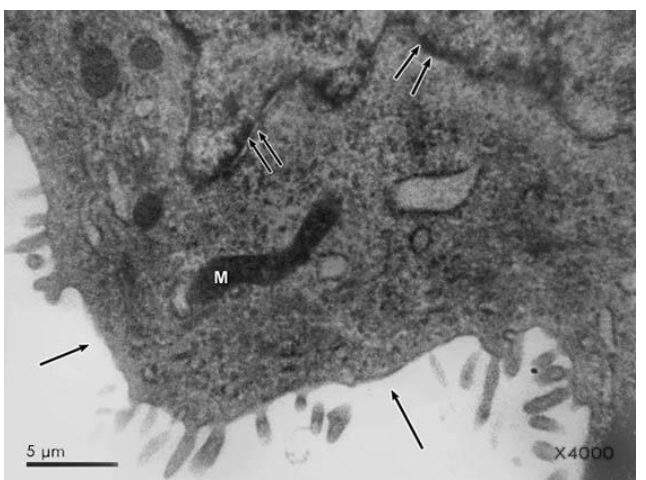

Figure 17: Electron micrograph of a portion of a villous from preeclamptic placenta 14 days showing local loss of microvilli on the free syncytial surface $(\uparrow)$ and irregular nucleus contour $(\uparrow \uparrow)$. Notice electron dense swollen mitochondria(M) .X4000.

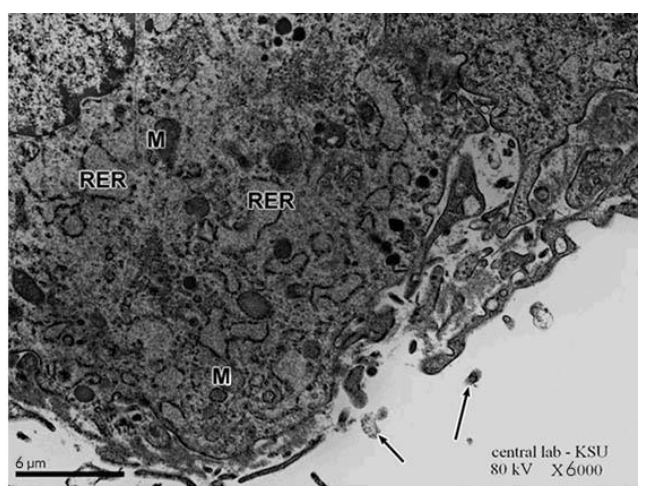

Figure 18: Electron micrograph of a portion of a villous from preeclamptic placenta 14 days showing minimally dilated sacs of R.E.R. The syncytial covering is reduced with loss of microvilli leaving only debris like layer $(\uparrow)$. The syncytial layer contains two types of mitochondria (M) which differ in electron density. X6000.

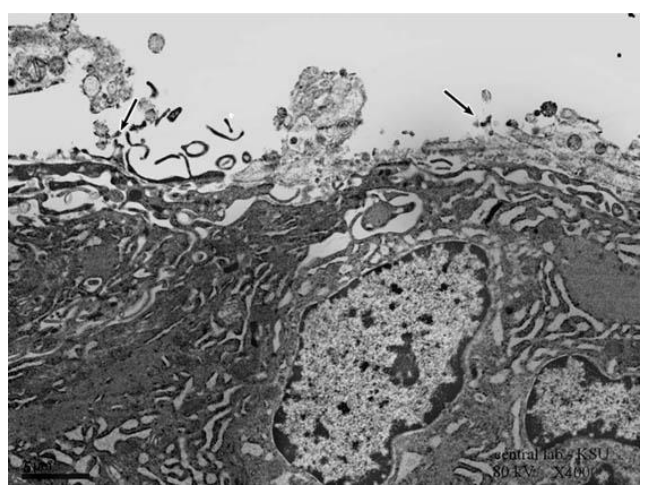

Figure 19: Electron micrograph of a portion of a villous from preeclamptic placenta (14 days) showing reduced and degenerated microvilli of syncytiotrophoblast $(\uparrow) . X 4000$.

and the placenta becomes increasingly oxygenated with time [14]. Normally, failure of conversion of spiral arterioles results in persistent placental hypoxia, maternal endothelial dysfunction, and placental insufficiency that lead to the clinical syndrome of preeclampsia [12]. In our significant electron microscopic observations, we provide further insight and analyzed the structural integrity of the STB layer in pre- eclamptic rats by transmission and scanning electron microscope. Our electron microscopic examination revealed destructed apical villi (brush border) with signs of apoptosis as increased cytoplasmic vacuoles and irregular contour of the nucleus (Figures 9,17 and 18). These changes were in harmony with those described by [15]. The authors studied the factors of preeclampsia in vitro and found that hypoxia and reperfusion could induce apoptosis in the syncytiotrophoblasts.

Ultrastructurally, it has been shown via electron microscopy that

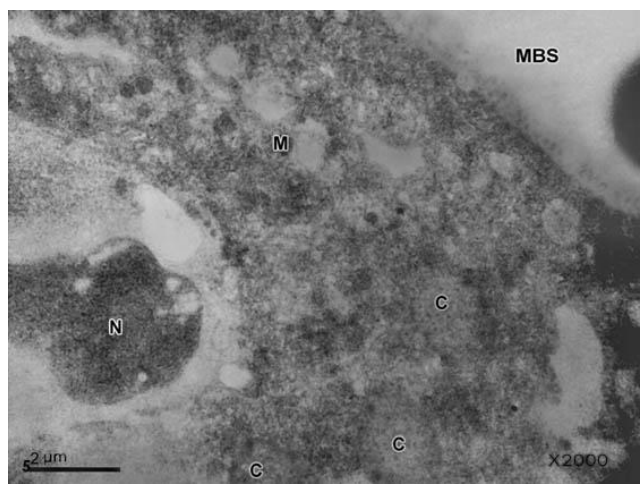

Figure 20: Electron micrograph of a portion of a villous from preeclamptic placenta (14 days) showing dense deposition of collagen fibers(C). Notice the nucleus with intranulear inclusions $(\mathrm{N})$.X2000.

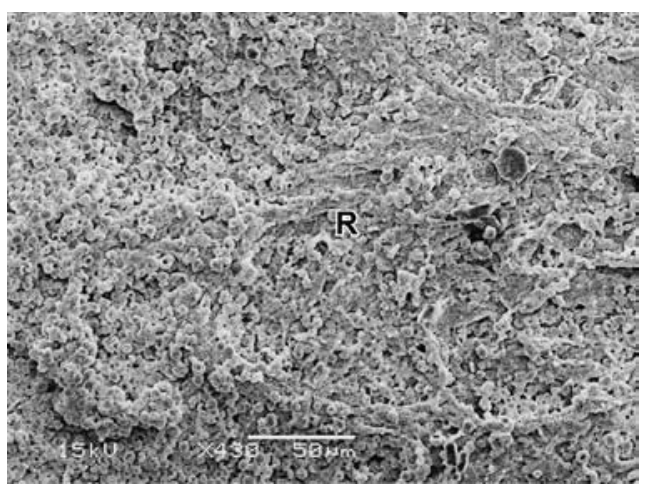

Figure 21: A scanning electron micrograph of chorionic villi of pre-eclamptic placenta, showing the framework of preeclamptic villi become irregular, rough and thickened due to fibrin deposition. ( $\times 430)$.

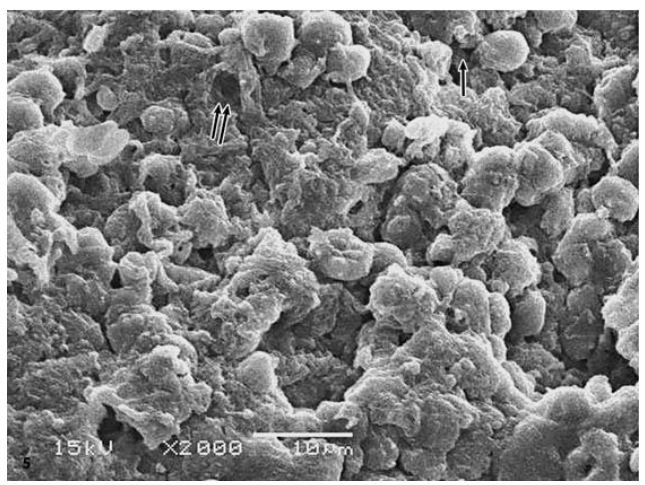

Figure 22: A scanning electron micrograph of chorionic villi of the preeclamptic placenta showing apparently the villi with infarcts $(\uparrow)$ and abruptions $(\uparrow \uparrow)$ $(\times 2000)$. 
Citation: Selim ME, Elshmry NG, Rashed EHA (2013) Electron and Scanning Microscopic Observations on the Syncytiotrophoblast Microvillous Membrane Contribution to Preeclampsia in Early Placental Rats. J Blood Disorders Transf 4:137. doi:10.4172/2155-9864.1000137

Page 7 of 8

there is severe alteration in pre-eclamptic group when compared to control group. In comparison, the study went a step further by including pre-eclamptic hypertension, increased albumin levels, decreased pup weights, creatine clearance and urine volume as well as shown in (Figures 1-5). Other than the apoptotic signs seen in the syncytial layer, there was an apparent damage in the mitochondria and the microvilli of the syncytial layer (Figures 14,18) in pre-eclamptic placenta, a finding similar to that of De Luca Brunori et al. [15]. In this study, mitochondrial degenerative changes occur in syncytiotrophoblast cells under doing apoptosis. Chronic hypoxia and alternate periods of hypoxia/reoxygenation within intervillous space is expected to trigger tissue oxidative stress and increase placental apoptosis [16]. Mitochondria contain the enzymatic systems which generates ATP through the Krebs cycle and oxidative phosphorylation. The formation of ATP requires oxygen which diminishes in preeclampsia. In this condition, it is suggested that syncytiotrophoblast cells undergo apoptotic events and fragments or debris are delivered into the intervillous space [17]. Our results are congruent with the authors [18] as they also found swollen and degenerating mitochondria in their model of hypoxic dual in vitro placental perfusion.

In our investigation, the observation of increased signs of apoptosis and cell damage together with the presence of intracellular oedema (Figure 11) in the syncytial layer is in line with the concept that there is more syncytial debris (Figure 13) shed into maternal space in preeclampsia [19], as apoptosis and cell damage proceeds the shedding of syncytial fragments into maternal lacunae or intervillous space. An increased release of syncytiotrophblasts microparticles (STBM) formed by plasma membrane blebbing during apoptosis are triggered in excess into the maternal circulation [20]. These detached fragments, debris of trophoblast, microvilli from syncytium or sycytial microparticles could be considered as causing endothelial damage among other factors.

Syncytiotrophoblastic detritus detached from the trophoblastic surface and resulting from a continuous process of regeneration, reparation or apoptosis are increased during preeclampsia.

Being a consequence of a poor blood supply subsequent to a spiral artery failure occurring in placental implantation, it constitutes the stimulus for a permanent inflammatory response characteristic in this common and dangerous pregnancy complication [21]. The authors also added that mitochondrial discharge cytochrome $c$; this protein affects the system of caspases and the trophoblast die by apoptosis. Longitudinal growth of the capillaries within the mature intermediate villi exceeds that of the villi themselves, so the capillaries coil and form loops that bulge from the villous surface, forming grapelike outgrowths known as terminal villi [21]. Their capillary convolutes from a rich network of loops with many intercalated branches and random focal dilatation known as sinusoids $[21,22]$. The development of this low impedance capillary network parallels the proportional rise in fetal cardiac output entering umbilical arteries to about $40 \%$ at term [23]; this arrangement favors maximum nutrient and gas exchange as the fetus grows. In our study the scanning microscopic examination revealed that irregular chorionic villi of the preeclamptic placenta were manifested (Figure 21) as compared with those of the normal placentas (Figure 8). Aberrantly many abruptions and multiple infarcts (Figure 22) were seen in pre-eclamptic specimens which is consistent with the hypoxia theory. Our results were in harmony with Zhang et al. [24] who performed a placental examination for changes with preeclampsia, and reported poor correlation between these changes with the clinical manifestations of pregnancy induced hypertension. The histological changes and clinical manifestation were also reported in a review article by Guller Seth [20] who explained how hypoxia/ reperfusion injury disrupts the syncytial architecture and results in the previously mentioned changes. The affected chorionic villi in our study also showed more roughened fibrillar skeleton with increased fibrin deposition (Figure 21). The same results were reported by Guller Seth [20] and Salafia et al. [25] who revealed that there were aberrantly high levels of intervillous fibrin which is a critical component of physiological repair and differentiation of the placental villi [26].

In view of these findings, we can point out that in preeclampsia the syncytiotrophoblast undergo apoptosis caused by mitochondrial damage, transforming it in a tissue with tendency to be fragmented. Such detritus detached from their surface could become the stimulus for endothelial dysfunction during the maintenance of the analyzed pregnancy complication.

\section{References}

1. Marín R, Gorostidi M, Portal CG, Sánchez M, Sánchez E, et al. (2000) Longterm prognosis of hypertension in pregnancy. Hypertens Pregnancy 19: 199209

2. Roberts JM, Lain KY (2002) Recent Insights into the pathogenesis of preeclampsia. Placenta 23: 359-372.

3. Hung TH, Skepper JN, Burton GJ (2001) In vitro ischemia-reperfusion injury in term human placenta as a model for oxidative stress in pathological pregnancies. Am J Pathol 159: 1031-1043.

4. Hung TH, Skepper JN, Charnock-Jones DS, Burton GJ (2002) Hypoxiareoxygenation: a potent inducer of apoptotic changes in the human placenta and possible etiological factor in preeclampsia. Circ Res 90: 1274-1281.

5. Sargent IL, Germain SJ, Sacks GP, Kumar S, Redman CW (2003) Trophoblas deportation and the maternal inflammatory response in pre-eclampsia. J Reprod Immunol 59: 153-160.

6. Redman CW, Sargent IL (2000) Placental debris, oxidative stress and preeclampsia. Placenta 21: 597-602

7. Allaire AD, Ballenger KA, Wells SR, McMahon MJ, Lessey BA (2000) Placenta apoptosis in preeclampsia. Obstet Gynecol 96: 271-276.

8. Rich BJ, Harbenson S, Moon DH (1984) Inhibition of aminopeptidases by bestatin derivatives. J Med CHem 27: 423-429.

9. Lenfant C; National Education Program Working Group on High Blood Pressure in Pregnancy (2001) Working group report on high blood pressure in pregnancy. J Clin Hypertens (Greenwich) 3: 75-88.

10. Brosens IA, Robertson WB, Dixon HG (1972) The role of the spiral arteries in the pathogenesis of preeclampsia. Obstet Gynecol Annu 1: 177-191.

11. Robertson WB, Brosens I, Dixon HG (1967) The pathological response of the vessels of the placental bed to hypertensive pregnancy. J Pathol Bacteriol 93 581-592.

12. Mutter WP, Karumanchi SA (2008) Molecular mechanisms of preeclampsia Microvasc Res 75: 1-8

13. Zhou Y, Bellingard V, Feng KT, McMaster M, Fisher SJ (2003) Human cytotrophoblasts promote endothelial survival and vascular remodeling through secretion of Ang2, PIGF, and VEGF-C. Dev Biol 263: 114-125.

14. Genbacev O, Zhou Y, Ludlow JW, Fisher SJ (1997) Regulation of human placental development by oxygen tension. Science 277: 1669-1672.

15. de Luca Brunori I, Battini L, Brunori E, Lenzi P, Paparelli A et al. (2005) Placental barrier breakage in preeclampsia: ultrastructural evidence. Eur Obstet Gynecol Reprod Biol 118: 182-189.

16. Grill S, Rusterholz C, Zanetti-Dällenbach R, Tercanli S, Holzgreve W, et al. (2009) Potential markers of preeclampsia--a review. Reprod Biol Endocrinol 7: 70 .

17. Castejón SOC, López AJ, Castejón MOC (2008) Cambios ultraestructurales del trofoblasto en los casos de hypoxia durante la preeclampsia. Rev Obstet Ginecol Venez 68: 168-174

18. Bachmaier N, Linnemann K, May K, Warzok R, Kuno S, et al. (2007) 
Citation: Selim ME, Elshmry NG, Rashed EHA (2013) Electron and Scanning Microscopic Observations on the Syncytiotrophoblast Microvillous Membrane Contribution to Preeclampsia in Early Placental Rats. J Blood Disorders Transf 4:137. doi:10.4172/2155-9864.1000137

Ultrastructure of human placental tissue after $6 \mathrm{~h}$ of normoxic and hypoxic dual in vitro placental perfusion. Placenta 28: 861-867.

19. Redman CW, Sargent IL (2008) Circulating microparticles in normal pregnancy and pre-eclampsia. Placenta 29: S73-77.

20. Guller S (2009) Role of the syncytium in placenta-mediated complications of preeclampsia. Thromb Res 124: 389-392.

21. Kaufmann P, Bruns U, Leiser R, Luckhart M, Winterhager E (1985) The fetal vascularization of term human placentral villi, II: intermediate and terminal villi. Anat Embryol 173: 203-214.

22. Kaufmann P, Burton G (1994) Anatomy and genesis of the placenta. In: Knobil E, Neill JD (eds.). The physiology of reproduction. New York: Raven Press $441-483$
23. Eik-Nes S, Brubakk AO, Ulstein M (1980) Measurement of human fetal blood flow. BMJ 1: 283-284.

24. Zhang P, Schmidt M, Cook L (2006) Maternal vasculopathy and histologic diagnosis of preeclampsia: poor correlation of histologic changes and clinical manifestation. Am J Obstet Gynecol 194: 1050-1056.

25. Salafia CM, Pezzullo JC, Minior VK, Divon MY (1997) Placental pathology of absent and reversed end-diastolic flow in growth-restricted fetuses. Obstet Gynecol 90: 830-836.

26. Humphrey RG, Smith SD, Pang L, Sadovsky Y, Nelson DM (2005) Fibrin enhances differentiation, but not apoptosis, and limits hypoxic injury of cultured term human trophoblasts. Placenta 26: 491-497. 